\title{
Classification of Chronic Kidney Disease in the Elderly: Pitfalls and Errors
}

\author{
Christopher G. Winearls ${ }^{\mathrm{a}}$ Richard J. Glassock ${ }^{\mathrm{b}}$ \\ ${ }^{a}$ Oxford Kidney Unit, The Churchill Hospital, Oxford Radcliffe Hospitals NHS Trust, Oxford, UK; ${ }^{b}$ David Geffen School \\ of Medicine at UCLA, Los Angeles, Calif., USA
}

Key Words

Ageing $\cdot$ Chronic kidney disease $\cdot$ Staging

\begin{abstract}
The average glomerular filtration rate (GFR) is lower in the elderly than in the young and is usually a consequence of biological ageing, the rate of which varies between individuals. In some subjects, the decline is aggravated by concomitant vascular disease. The prevalence of significant kidney disease in the elderly has been overestimated - largely by rendering a diagnosis of chronic kidney disease by reference to estimates of GFR which are found in the young. A stable low GFR in the elderly, provided it is physiologically sufficient to meet homeostatic demands, is not a disease per se and seldom progresses to true kidney failure. However, it can be a risk factor for acute kidney injury drug misdosing, and possibly cardiovascular disease, so it should be noted.
\end{abstract}

Copyright $\odot 2011$ S. Karger AG, Basel

\section{Introduction}

Almost all of the manifestations of kidney disease are more common in elderly and very old (note: for the purposes of this article, the elderly are those subjects between retirement age and average life span, i.e. between 65 and
80 years of age. Those over 80 years might reasonably be called 'very old') subjects who represent an increasing proportion of the population (16\% in the United Kingdom). They are at greater risk, and have a higher incidence and prevalence of both of the extreme problems acute kidney injury (AKI) and end-stage renal disease (ESRD) requiring renal replacement therapy (RRT) $[1,2]$. In 2008, in England, the incidence of new RRT patients (i.e. treated ESRD, was 150 pmp for the age group 45-64 years and $325 \mathrm{pmp}$ for those $>65$ years [3]).

The precursor state of ESRD, namely 'generic chronic kidney disease (CKD)', as currently defined, is also much more common in the elderly and very old. So, if the extreme problems are to be prevented or avoided, it is logical to identify those with true CKD who are at a clinically meaningful enhanced risk of adverse outcomes. This would be especially true if safe and effective interventions were available to mitigate the risk associated with CKD. The current classification system will do so, but like a fishing trawler it captures many more innocent subjects than it should. Estimates of the prevalence of CKD in the elderly generated by the current CKD definition and staging systems are so high as to appear unrealistic. 25$35 \%$ of subjects over the age of 65 years of age, only a minority of whom have abnormal proteinuria, meet the current criteria for a diagnosis of CKD [reviewed in ref. 4]. Of these, fewer than $0.1 \%$ will develop ESRD each year.

\section{KARGER}

Fax +4161306 1234 E-Mail karger@karger.ch www.karger.com

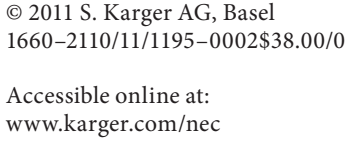




\section{Understanding CKD in the Elderly}

Why Is This Imbalance So Evident and What Can We Do to Better Understand and Correct It?

The first problem is that of the definition of abnormal. One way to define a glomerular filtration rate (GFR) as being abnormally low in any subject, young or old, is that it is lower than it should be. As with any biological variable, one could rely on the distribution of GFR in the healthy population and define an abnormal, disease-designating GFR as $<5$ th percentile at any given age (i.e. the chance of this GFR being this low by chance is $<1 / 20$ ). The question then is whether this low(er) GFR is a consequence of kidney pathology that might progress if left unattended. If it is a consequence of pathology, then one can say the patient has CKD, with the disease having this degree of impact on overall kidney function. Then there is the issue of whether the GFR, although low, is sufficient or insufficient for homeostasis. The objective evidence for insufficiency of GFR and overall kidney function would be abnormal sodium balance causing hypertension, a blunted erythropoietin axis causing anaemia, disturbed calcium and phosphate balance causing secondary hyperparathyroidism. Homeostatic failure seems to occur with increasing frequency at a GFR $<60$ $\mathrm{ml} / \mathrm{min} / 1.73 \mathrm{~m}^{2}$ and most obviously at $<45 \mathrm{ml} / \mathrm{min} /$ $1.73 \mathrm{~m}^{2}[5]$.

The current staging system for CKD is largely based on the notion that a GFR $>90 \mathrm{ml} / \mathrm{min} / 1.73 \mathrm{~m}^{2}$ is nor$\mathrm{mal} ; 60-89 \mathrm{ml} / \mathrm{min} / 1.73 \mathrm{~m}^{2}$ is reduced and $<60 \mathrm{ml} / \mathrm{min} /$ $1.73 \mathrm{~m}^{2}$ is always abnormal, irrespective of the presence of corroboratory evidence of kidney damage [6]. This is a rigid approach which ignores biological variability, redundancy and renal reserve. These values are valid for measured GFR (mGFR) in young adults but do not match the findings in healthy individuals across the age range. Cross-sectional measurements of mGFR and estimated GFR (eGFR) in healthy individuals, e.g. potential kidney donors, show that there is a reduction in the mean value of $6-8 \mathrm{ml} / \mathrm{min} / 1.73 \mathrm{~m}^{2}$ per decade such that by these criteria the majority of subjects $>40$ years of age would be considered to have 'reduced kidney function' $[7,8]$.

It is therefore inappropriate to diagnose CKD on GFR values alone, especially in the elderly. The diagnosis should require that the GFR be inappropriately low for that individual and a consequence of a plausible pathology. Additional criteria for diagnosing kidney disease would be a more rapid decline in GFR than is usually observed with normal ageing $\left(>1.0 \mathrm{ml} / \mathrm{min} / 1.73 \mathrm{~m}^{2} /\right.$ year $)$ and the presence of abnormal (overt) proteinuria or albu- minuria. Many of the elderly currently diagnosed as having CKD would not meet these criteria. The importance of proteinuria has recently been emphasized by Hallan and Orth [9] in this journal and Tonelli et al. [10].

The second problem is the reproducibility and consistency of eGFR. Apart from the tendency of all estimating formulas to underestimate the GFR when it is nearer to the physiological range, it can vary without obvious reason $[11,12]$. The CKD-EPI formula estimates mGFR with greater accuracy than other estimating formulas, especially in the physiological range, but we do not yet have a nomogram of the measurement in large numbers of healthy individuals across the age spectrum $20-80$ years [13].

The third problem is elevating 'micro-albuminuria', expressed as an albumin-to-creatinine ratio of 30-300 $\mathrm{mg} / \mathrm{g}$ in a 'spot' urine sample, to the rank of a stand-alone criterion for the diagnosis of CKD. This creates a very low threshold for diagnosing a CKD because 'micro-albuminuria', so defined, can wax and wane, be caused by systemic endothelial injury which occurs in hypertension, cigarette smoking, occult and overt inflammation and obesity. The kidney registers the injury but is not the prime target of it. Using urinary creatinine as the denominator for the ratio will increase it in the elderly who have a lower muscle mass and creatinine excretion. A 'normal' urine albumin excretion rate may therefore be reported as an abnormal ratio [14].

In practice many physicians apply a 'one-strike' policy and allocate patients to a CKD stage because of a single 'abnormal' eGFR or urine albumin-to-creatinine ratio without observing the 3 -month persistence rule. If it were scrupulously obeyed, the reported prevalence of CKD would be much lower, by as much as $30 \%$ [15].

One can test whether the CKD staging schema currently in wide use is appropriate by asking whether individuals allocated to the same stage of CKD have more or less similar outcomes irrespective of age? Since the majority of cases of CKD are in stage 3 and the majority of these are elderly, the best test would be a comparison of the risk of developing CKD stage 5, AKI and death attributable to $\mathrm{CKD}$, in young or elderly subjects. While such stratification has been examined on a categorical basis (age $>65$ or $<65$ years), we still are uncertain of comparable information for the very old [16]. The recent data from the PREVEND study is informative. Measures of GFR were independently and significantly associated with cardiovascular events only in subjects $<60$ years of age [17]. 


\section{GFR and Pathology in Ageing}

There is an additional fundamental and probably Delphic question as to whether the generally observed reduction in GFR in the elderly is a consequence of pathological processes in the absence of which GFR would be similar in the young and the elderly [reviewed in ref. 18]. Intuitively, and by analogy with almost every other organ system, one would predict that there would be an age-related reduction in kidney function with time. There are morphological and functional differences in the kidneys of the elderly compared to the young, which are explained in part by differences in their vasculature. Since this is a general phenomenon in all circulations and elderly subjects and is seen in laboratory animals, it is a moot point as to whether this represents a natural process of tissue and organ senescence or a form of pathology.

One should nevertheless take a common sense clinical attitude to the reduced (compared to younger subjects) GFRs of the elderly. Just because they do not deserve the label of CKD does not mean that they should not be handled with care. A low GFR may be a consequence of se- nescence, subclinical vascular disease and genuine pathology (sometimes occurring simultaneously). The clinician should not be tempted to use age as an excuse not to consider the possibility of bona-fide renal disease which is much more likely to progress or cause untoward events such as AKI or cardio-vascular disease.

A low GFR implies a reduced reserve and vulnerability to drug overdosing (especially with water-soluble drugs that depend on GFR for excretion), and the insults which can precipitate AKI. The eGFR can be a guide to risk in the individual without the need to describe the individual as chronically diseased. Risks and diseases are different [19].

The elderly and the very old deserve careful analysis before they are labelled as having a chronic disease based solely on a single laboratory measurement, especially one with inherent inaccuracies.

\section{Disclosure Statement}

The authors have no conflicts of interest to declare.

\section{References}

1 Hsu CY: Where is the epidemic in kidney disease? J Am Soc Nephrol 2010;21:16071611.

-2 Hsu CY, McCulloch CE, Fan D, Ordonez JD, Cherow GM, Go S: Community-based incidence of acute renal failure. Kidney Int 2007; 72:208-212.

3 Byrne C, Ford D, Gilg J, Ansell D, Feehally J: UK ESRD Incident rates in 2008: national and centre-specific analyses. UK Renal Registry 12th Annual Report, 2009. Bristol, UK Renal Registry.

4 James MT, Hemmelgarn BR, Tonelli M: Early recognition and prevention of chronic kid ney disease. Lancet 2010;375:1296-1309.

5 Moranne O, Froissart M, Rossert J, et al: Timing of onset of CKD-related metabolic complications. J Am Soc Nephrol 2009;20: 164-171.

6 K/DOQI clinical practice guidelines for chronic kidney disease: evaluation, classification, and stratification. Am J Kidney Dis 2002;39:S1-S266.

$\checkmark 7$ Rule AD, Amer H, Cornell LD, et al: The association between age and nephrosclerosis on renal biopsy among healthy adults. Ann Intern Med 2010;152:561-567.

$>8$ Wetzels JF, Kiemeney LA, Swinkels DW, Willems HL, Heijer MD: Age- and gender- specific reference values of estimated GFR in Caucasians: The Nijmegen Biomedical Study. Kidney Int 2007;72:632-637.

$\checkmark 9$ Hallan IS, Orth SR: The conundrum of chronic kidney disease classification and end-stage renal risk prediction in the elderly - what is the right approach? Nephron Clin Pract 2010;116:c307-c316.

10 Tonelli $\mathrm{M}$ et al., for the Alberta Kidney Disease Network: Using proteinuria and estimated glomerular filtration rate to classify risk in patients with chronic kidney disease. Ann Intern Med 2011;154:12-21.

11 Froissart M, Rossert J, Jacquot C, Paillard M, Houillier P: Predictive performance of the modification of diet in renal disease and Cockcroft-Gault equations for estimating renal function. J Am Soc Nephrol 2005;16: 763-773.

12 Tent H, Rook M, Stevens LA, et al: Renal function equations before and after living kidney donation: a within-individual comparison of performance at different levels of renal function. Clin J Am Soc 2010;5:19601968.

13 Stevens LA et al: Comparative performance of the CKD Epidemiology collaboration (CKD EPI) and the Modification of Diet in Renal Disease (MDRD) study equations for estimating GFR levels above $60 \mathrm{ml} / \mathrm{min} /$ $1.73 \mathrm{~m}^{2}$. Am J Kidney Dis 2010;56:486-495.

14 Ellam TJ, El Nahas M: Proteinuria thresholds are irrational: a call for proteinuria indexing. Nephron Clin Pract 2010;118:c217c224.

15 Eriksen BO, Ingebretsen OC: In chronic kidney disease staging the use of chronicity criterion affects prognosis and rate of progression. Kidney Int 2007;72:1242-1248.

16 Chronic Kidney Disease Prognosis Consortium: Association of estimated glomerular filtration rate and albuminuria with allcause and cardiovascular mortality in general population cohorts: a collaborative meta-analysis. Lancet 2010;375:2073-2081.

17 van der Velde M, Bakker SJL, de Jong PE, Gansevoort RT: Influence of age and measure of eGFR on the association between renal function and cardiovascular events. Clin J Am Soc Nephrol 2010;5:2053-2059.

18 Glassock RJ, Winearls C: Ageing and the glomerular filtration rate: truths and consequences. Trans Am Clin Climatol Assoc 2009; 120:419-428.

19 Clase CM Garg AX, Kibberd BA: Classifying kidney problems: can we avoid framing risks as diseases? Br Med J 2004;329:912915. 